\title{
Nonadherence to Statins and Antihypertensives and Hospitalizations Among Elderly Medicare Beneficiaries With Incident Cancer
}

\author{
Ishveen Chopra, MS, MBA, PhDa; Nilanjana Dwibedi, MBA, PhD; ; Malcolm D. Mattes, $\mathrm{MD}^{\mathrm{b}}$; \\ Xi Tan, PharmD, PhD; ; Patricia Findley, DrPH, MSW, LCSW ${ }^{c}$; and Usha Sambamoorthi, $\mathrm{PhD}^{\text {a }}$
}

\begin{abstract}
Background: Incident cancer diagnosis may increase the risk of coronary artery disease (CAD)-related hospitalizations, especially in older individuals. Adherence to statins and/or angiotensin-converting enzyme inhibitors (ACEIs)/angiotensin II receptor blockers (ARBs)/ $\beta$-blockers reduces CAD-related hospitalizations. This study examined the relationship between medication adherence and CAD-related hospitalizations immediately following cancer diagnosis. Patients and Methods: A retrospective observational longitudinal study was conducted using SEER-Medicare data. Elderly Medicare fee-for-service beneficiaries with preexisting CAD and incident breast, colorectal, or prostate cancer $(\mathrm{N}=12,096)$ were observed for 12 months before and after cancer diagnosis. Hospitalizations measured every 120 days were categorized into CAD-related hospitalization, other hospitalization, and no hospitalization. Medication adherence was categorized into 5 mutually exclusive groups: adherent to both statins and ACEIs/ARBs/ $\beta$-blockers (reference group), not adherent to both statins and ACEIs/ ARBs/ $\beta$-blockers, adherent to either statins or ACEIs/ARBs/ $\beta$-blockers, use of one medication class and adherent to that class, and use of one medication class and not adherent to that class. The relationship between medication adherence and hospitalization was analyzed using repeated measures multinomial logistic regressions. Inverse probability treatment weights were used to control for observed group differences among medication adherence categories. Results: Adherence to both statins and ACEIs/ARBs/ $\beta$-blockers was estimated at $31.2 \%$ during the 120-day period immediately following cancer diagnosis; $13.7 \%$ were not adherent to both medication classes during the same period, and $27.4 \%$ had CAD-related hospitalizations immediately after cancer diagnosis, which declined to $10.6 \%$ during the last 4 months of the postdiagnosis period. In the adjusted analyses, those not adherent to both statins and ACEIs/ARBs/ $\beta$-blockers were more likely to have CAD-related hospitalization compared with those adherent to both medication classes (adjusted odds ratio, 1.82; $95 \%$ Cl, 1.72-1.92; $P<.0001)$. Conclusions: Given the complexity of interaction between CAD and cancer, it is important to routinely monitor medication adherence in general clinical practice and to provide linkages to support services that can increase medication adherence.
\end{abstract}

J Natl Compr Canc Netw 2017;15(11):1351-1360 doi: 10.6004/jnccn.2017.0168

Coronary artery disease (CAD) remains the leading cause of hospitalization in the United States, although the rate of hospitalization for CAD has declined from $77 \%$ in 2000 to $44 \%$ in $2010 . .^{1}$ This decline may be due to control of risk factors, including serum cholesterol

From the Departments of aPharmaceutical Systems and Policy, West Virginia University School of Pharmacy, and ' ${ }^{\mathrm{R}}$ Radiation Oncology, West Virginia University School of Medicine, Morgantown, West Virginia; and 'School of Social Work, Rutgers University, New Brunswick, New Jersey. Submitted January 27, 2017; accepted for publication June 7, 2017. The authors have disclosed that they have no financial interests, arrangements, affiliations, or commercial interests with the manufacturers of any products discussed in this article or their competitors.

Research reported in this publication was supported by the National Institute of General Medical Sciences of the NIH, award number levels, hypertension, and smoking; increased adoption of evidence-based medication use and timeliness of diagnosis and treatment of patients with CAD have also contributed to the decline in hospitalizations., ${ }^{2,3}$ According to the Healthcare Cost and Utilization Project
U54GM104942. The content is solely the responsibility of the authors and does not necessarily represent the official views of the NIH.

Author contributions: Study design: Chopra, Sambamoorthi. Data analysis: Chopra, Sambamoorthi. Implementation: Chopra. Manuscript preparation: Chopra. Critical review: Sambamoorthi. Feedback on manuscript: Dwibedi, Mattes, Tan, Findley.

Correspondence: Nilanjana Dwibedi, MBA, PhD, Department of Pharmaceutical Systems and Policy, West Virginia University, School of Pharmacy, Robert C. Byrd Health Sciences Center, PO Box 9510, Morgantown, WV 26506-9510. E-mail: nidwibedi@hsc.wvu.edu 
(HCUP), CAD accounted for inpatient expenditures of nearly $\$ 9.5$ billion (2010). ${ }^{4}$ The per-capita hospital expenditures were estimated at \$22,700 in 2014 , accounting for approximately $62 \%$ of total direct healthcare expenditures, ${ }^{5,6}$ suggesting that hospitalizations are the primary drivers of total direct medical care expenditures.

Existing evidence also suggests that individuals aged $\geq 65$ years with incident cancer may be at risk for non-cancer-related hospitalizations. Although not specific to CAD-related hospitalizations, men with incident prostate cancer and cardiometabolic conditions were more likely to experience hospitalization in the period immediately after cancer diagnosis. ${ }^{7}$ The risk of first hospitalization for CAD increases during the 6 months after cancer diagnosis, ${ }^{8}$ perhaps due to cardiotoxicity associated with cancer treatments, such as radiotherapy, chemotherapy, and hormone therapy. ${ }^{9-11}$ Some studies have reported an increased risk of cardiovascular events, complications, and mortality in women who were treated with radiotherapy ${ }^{12-14}$ or were exposed to cardiotoxic drugs (trastuzumab and anthracycline). ${ }^{15-17}$

Adherence to angiotensin-converting enzyme inhibitors (ACEIs), angiotensin II receptor blockers (ARBs), $\beta$-blockers, and statins have been shown to not only improve survival, ${ }^{18,19}$ but also to reduce the risk of CAD-related hospitalizations. ${ }^{18,20}$ Specifically, statins have been reported to reduce cancerspecific mortality in those with breast, colorectal, and prostate cancers, ${ }^{21-24}$ suggesting that adherence to statins may be even more important for patients with cancer.

However, to date, no study has examined the relationship between adherence to statins and ACEIs/ $\mathrm{ARBs} / \beta$-blockers, and CAD-related hospitalizations in older individuals (age $\geq 65$ years) with preexisting CAD and incident cancer. Because CAD is the most common preexisting condition among older adults (age $\geq 65$ years) diagnosed with cancer, ${ }^{25-27}$ it is important to analyze the relationship between adherence to both statins and ACEIs/ARBs/ $\beta$-blockers, and CAD-related hospitalizations among patients with cancer. Therefore, we evaluated the relationship between adherence to both statins and ACEIs/ $\mathrm{ARBs} / \beta$-blockers, and CAD-related hospitalizations among elderly fee-for-service (FFS) Medicare beneficiaries with preexisting CAD and incident breast, colorectal, or prostate cancer, after controlling for cardiotoxic cancer treatments and other risk factors that may affect CAD-related hospitalizations.

\section{Patients and Methods}

\section{Conceptual Framework}

This study adapted Andersen's Behavioral Model of Health Services Utilization to select independent variables that may influence CAD-related hospitalizations (health outcome). ${ }^{28,29}$ The sixth iteration of Andersen's model posits that predisposing (eg, age, sex, race/ethnicity), enabling (eg, education, poverty status), and need factors (eg, chronic conditions; cancer type, stage, and treatment; CAD severity), and external environment affect health behavior or healthcare use, which in turn affects health outcomes. Because these factors affect both healthcare use and health outcomes, they were included as covariates in the analysis.

\section{Study Design}

The study used a retrospective observational longitudinal cohort design with baseline (24-36 months before cancer diagnosis), prediagnosis (12 months before cancer diagnosis), and postdiagnosis (12 months after cancer diagnosis) periods.

\section{Data Sources}

Data were obtained from the SEER registries, Medicare claims, the American Community Survey (ACS), and the Area Health Resource Files (AHRF). The SEER program is an epidemiologic surveillance system consisting of population-based tumor registries that collects data on all incident cases of cancer that occur in persons residing in 18 SEER areas. ${ }^{30}$ The ACS census tract files provided information on the census tract poverty status, ${ }^{31}$ and the AHRF was used to derive county-level information. ${ }^{32}$

\section{Study Population}

Individuals with CAD were identified using a validated algorithm developed by the Centers for Medicare $\&$ Medicaid Services (CMS) ${ }^{33}$; the algorithm used ICD-9-CM codes. Individuals with at least 1 inpatient or 2 outpatient claims (institutional outpatient, physician office, and home health agency claims) with a primary or secondary diagnosis of CAD during the baseline period were classified as 
having preexisting CAD. Individuals with incident cancer were identified from the SEER registry for the period between January 2008 and December 2011.

Other inclusion criteria were age $\geq 68$ years at cancer diagnosis, no missing data for type and stage of cancer, alive with continuous FFS Medicare Parts $A$ and $B$ enrollment during the entire study period, continuous Part D enrollment during the prediagnosis and postdiagnosis period, no missing information on county, and having $\geq 2$ prescriptions for either statins or $\geq 2$ prescriptions for any one of the following medication types: ACEIs, ARBs, or $\beta$-blockers (Figure 1).

\section{Measures}

Dependent Variable: Any Hospitalization for CAD Events: The dependent variable was categorized into 3 mutually exclusive groups based on a hierarchy: any CAD-related hospitalization, other hospitalization, and no hospitalization. CAD-related hospitalizations were defined as inpatient admissions for any cardiovascular event (heart attack, heart failure, angina, or stroke) and were identified from primary and secondary diagnoses using ICD-9-CM codes. ${ }^{34}$ This measure was calculated every 120 days before cancer diagnosis and every 120 days during the postdiagnosis period. A 120-day interval before cancer diagnosis was a baseline measurement to ensure that the change in hospitalization was associated with adherence and not due to any other factors.

Key Independent Variable: Adherence to Statins, ACEIs/ARB/ $\beta$-blockers: Adherence was calculated 120 days before cancer diagnosis and every 120 days during the postdiagnosis period. A 120 day interval before cancer diagnosis was a baseline measurement to account for change in adherence due to cancer diagnosis. Based on the type of drug regimen, individuals were classified into 5 mutually exclusive groups: adherent to both medication classes (statins and ACEIs/ARBs/ $\beta$-blockers), not adherent to both medication classes, adherent to either medication class, use of one medication class and adherent, and use of one medication class and not adherent.

Medication classes were identified using the generic names in the Medicare Part D event files. Proportion of days covered (PDC) was used to measure adherence and was calculated for those who filled at least 2 prescriptions for these medications. The PDC was calculated as:

Days of medication supplied during each

$\frac{\text { time period }\left(t_{0}, t_{1}, t_{2}, t_{3}\right)}{\text { Days in each time period }(120 \text { days })}$

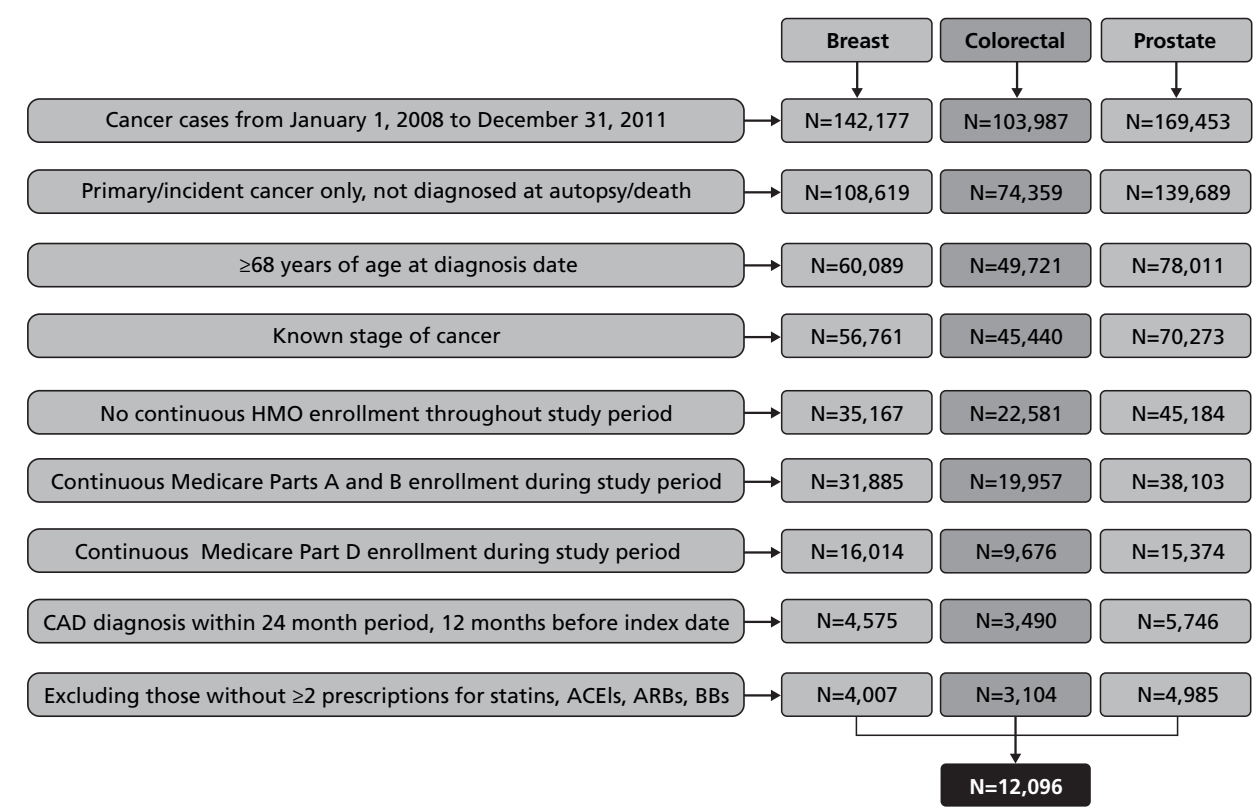

Figure 1. Schematic presentation of selection criteria for study cohort.

Abbreviations: ACEls, angiotensin-converting enzyme inhibitors; $A R B s$, angiotensin II receptor blockers; $B B, \beta$-blockers; CAD, coronary artery disease; HMO, Health Maintenance Organization. 
For those with a drug regimen, PDC includes days within a specific period when an individual is covered for both statins and $\beta$-blockers, ACEIs, or ARBs. PDC was dichotomized, wherein individuals with PDC $\geq 80 \%$ were considered as adherent. ${ }^{35}$

Other Independent Variables: Other independent variables included predisposing (age, sex, race/ethnicity), enabling (census tract education level and poverty status, marital status, Medicare Part D prescription drug coverage), need factors (chronic physical and mental health conditions, CAD severity, and cancer type, stage, and treatment), personal health practices (tobacco and alcohol use), healthcare use (visits to primary care/cardiologist), and external environment (SEER region, county metropolitan status, percent of cardiologists and oncologists in a county).

A proxy measure was used for CAD severity based on the CMS hierarchical condition category (HCC) classification system. Based on the CMS risk adjustment model, each of the HCC codes for CAD was assigned a specific score based on the risk and severity, ${ }^{36}$ ranging from 0.231 to 0.349 . Thus, higher scores represented more severe manifestations of CAD.

\section{Statistical Analyses}

Unadjusted subgroup differences in time-invariant characteristics between medication adherence categories were tested with chi-square statistics. Given that medication adherence was measured every 120 days during the prediagnosis and postdiagnosis periods, each individual had 4 observations. These 4 observations were not independent; therefore, we analyzed the relationship between adherence categories and CAD-related hospitalizations using multinomial logistic regression for repeated measures. Marginal models (eg, population-averaged models) were selected to describe changes in CAD-related hospitalization given changes in adherence categories, while accounting for nonindependence of observations within individuals. ${ }^{37}$

Inverse Probability Treatment Weight: As shown in supplemental eAppendix 1 (available online with this article at JNCCN.org), there were significant associations between medication adherence categories and for other independent variables. Therefore, we derived inverse probability treatment weights (IPTWs) to balance the independent variables using a multinomial logistic regression on medication adherence categories during the 120-day period immediately after cancer diagnosis to take advantage of the availability of cancer-related variables (eg, stage, type, treatment). These IPTWs derived for each individual were then used as weights in CAD-related hospitalization analyses.

\section{Results}

Study Population Characteristics: Before and After IPTW Adjustment

Table 1 summarizes the study population characteristics. The study population comprised 12,096 elderly FFS Medicare beneficiaries with preexisting CAD and incident cancer diagnosis. Table 2 summarizes the characteristics of the cohort by adherence to statins and/or ACEIs/ARBs/ $\beta$-blockers before and

\begin{tabular}{|c|c|c|}
\hline \multirow[b]{2}{*}{ Characteristics } & \multicolumn{2}{|c|}{ Total Population } \\
\hline & $\mathbf{N}$ & $\%$ \\
\hline \multicolumn{3}{|l|}{ Age, $y$} \\
\hline $68-70$ & 1,983 & 16.4 \\
\hline $71-74$ & 2,887 & 23.9 \\
\hline $75-79$ & 3,158 & 26.1 \\
\hline$\geq 80$ & 4,068 & 33.6 \\
\hline \multicolumn{3}{|l|}{ Race/Ethnicity } \\
\hline White & 9,928 & 82.1 \\
\hline African American & 1,013 & 8.4 \\
\hline Other & 1,155 & 9.5 \\
\hline \multicolumn{3}{|l|}{ Cancer diagnosis } \\
\hline Women with breast cancer & 4,007 & 33.1 \\
\hline Women with colorectal cancer & 1,705 & 14.1 \\
\hline Men with colorectal cancer & 1,399 & 11.6 \\
\hline Men with prostate cancer & 4,985 & 41.2 \\
\hline \multicolumn{3}{|l|}{ Cancer stage } \\
\hline $0 / 1$ & 3,565 & 29.5 \\
\hline II & 6,572 & 54.3 \\
\hline III/IV & 1,959 & 16.2 \\
\hline \multicolumn{3}{|l|}{ Cancer treatment } \\
\hline Cardiotoxic & 4,821 & 34.9 \\
\hline Non-cardiotoxic & 6,668 & 48.3 \\
\hline None & 2,322 & 16.8 \\
\hline \multicolumn{3}{|l|}{ SEER region } \\
\hline Northeast & 2,749 & 22.7 \\
\hline South & 2,977 & 24.6 \\
\hline North-Central & 1,691 & 14.0 \\
\hline West & 4,679 & 38.7 \\
\hline \multicolumn{3}{|l|}{ Index year } \\
\hline 2008 & 2,966 & 24.5 \\
\hline 2009 & 2,975 & 24.6 \\
\hline 2010 & 2,992 & 24.7 \\
\hline 2011 & 3,163 & 26.1 \\
\hline
\end{tabular}

Based on 12,096 elderly fee-for-service Medicare beneficiaries with preexisting coronary artery disease and incident breast, colorectal, and prostate cancers between 2008 and 2011. Table includes time-invariant characteristics. 
CAD Medication Adherence and Hospitalization

Table 2. Selected Characteristics by Adherence to Statins and/or ACEls/ARBs/ $\beta$-Blockers

\begin{tabular}{|c|c|c|c|c|c|c|c|c|c|c|c|c|}
\hline \multirow[b]{2}{*}{ Characteristics } & \multicolumn{6}{|c|}{ Before IPTW (\%) } & \multicolumn{6}{|c|}{ After IPTW (Weighted \%) } \\
\hline & $\begin{array}{l}\mathbf{A D H} \\
2 \mathbf{R x}^{\mathrm{a}}\end{array}$ & $\begin{array}{c}\text { No } A D H \\
2 \mathbf{R x}^{\mathrm{b}}\end{array}$ & $\begin{array}{c}\text { ADH } \\
1 \text { of } 2 R^{c}\end{array}$ & $\begin{array}{l}\text { ADH } \\
1 \mathbf{R x}^{d}\end{array}$ & $\begin{array}{c}\text { No ADH } \\
1 \mathbf{R x}^{\mathrm{e}}\end{array}$ & $\operatorname{Sig}^{f}$ & $\begin{array}{l}\text { ADH } \\
2 \mathbf{R x}^{\mathrm{a}}\end{array}$ & $\begin{array}{c}\text { No } A D H \\
2 \mathbf{R x}^{\mathrm{b}}\end{array}$ & $\begin{array}{c}\text { ADH } \\
1 \text { of } 2 R^{c}\end{array}$ & $\begin{array}{l}\text { ADH } \\
1 R^{d}\end{array}$ & $\begin{array}{c}\text { No }{ }_{\mathbf{R}} \mathbf{x}^{\mathrm{e}} \\
\end{array}$ & $\mathrm{Sig}^{\dagger}$ \\
\hline Age, y & & & & & & $\star * *$ & & & & & & NS \\
\hline $68-70$ & 18.0 & 13.7 & 17.1 & 14.9 & 16.3 & & 16.5 & 16.8 & 16.3 & 16.5 & 16.6 & \\
\hline $71-74$ & 25.7 & 23.3 & 24.9 & 22.0 & 21.1 & & 24.0 & 23.8 & 23.9 & 24.0 & 24.0 & \\
\hline $75-79$ & 27.3 & 24.6 & 27.2 & 25.9 & 23.4 & & 26.4 & 26.0 & 25.9 & 25.8 & 26.3 & \\
\hline$\geq 80$ & 29.0 & 38.4 & 30.8 & 37.2 & 39.2 & & 33.1 & 33.4 & 33.8 & 33.8 & 33.0 & \\
\hline Race/Ethnicity & & & & & & $* * *$ & & & & & & NS \\
\hline White & 84.5 & 77.6 & 82.7 & 82.4 & 79.6 & & 82.2 & 81.6 & 82.2 & 81.7 & 82.3 & \\
\hline African American & 6.4 & 10.8 & 7.6 & 8.8 & 11.2 & & 8.4 & 8.4 & 8.2 & 8.5 & 8.5 & \\
\hline Other & 9.1 & 11.6 & 9.8 & 8.8 & 9.3 & & 9.4 & 10.0 & 9.5 & 9.7 & 9.2 & \\
\hline Medicare Part D coverage & & & & & & $* * *$ & & & & & & NS \\
\hline Exceeded & 5.6 & 2.7 & 3.9 & 3.4 & 2.4 & & 4.1 & 4.3 & 4.0 & 4.1 & 3.8 & \\
\hline Not reached & 90.5 & 95.4 & 93.1 & 94.5 & 96.1 & & 93.2 & 93.1 & 93.3 & 93.1 & 93.4 & \\
\hline Reached & 3.8 & 1.9 & 3.0 & 2.1 & 1.5 & & 2.8 & 2.6 & 2.7 & 2.8 & 2.7 & \\
\hline Cancer diagnosis categories & & & & & & $* * *$ & & & & & & NS \\
\hline Women with breast cancer & 33.8 & 23.3 & 32.5 & 41.6 & 31.2 & & 34.0 & 33.5 & 33.2 & 33.5 & 33.5 & \\
\hline Women with colorectal cancer & 9.3 & 21.2 & 14.8 & 12.1 & 19.4 & & 13.7 & 13.9 & 14.1 & 13.8 & 13.7 & \\
\hline Men with colorectal cancer & 9.5 & 19.0 & 11.0 & 7.9 & 14.6 & & 11.3 & 11.4 & 11.4 & 11.7 & 11.4 & \\
\hline Men with prostate cancer & 47.4 & 36.5 & 41.7 & 38.4 & 34.8 & & 41.0 & 41.3 & 41.3 & 41.0 & 41.3 & \\
\hline Cancer stage & & & & & & $* * *$ & & & & & & NS \\
\hline $0 / 1$ & 29.2 & 25.9 & 30.3 & 32.6 & 27.9 & & 29.8 & 29.4 & 29.4 & 29.4 & 29.8 & \\
\hline II & 57.4 & 51.9 & 53.8 & 52.5 & 53.0 & & 54.0 & 55.0 & 54.4 & 54.3 & 54.1 & \\
\hline $\mathrm{III} / \mathrm{IV}$ & 13.4 & 22.1 & 15.8 & 14.9 & 19.1 & & 16.2 & 15.6 & 16.2 & 16.3 & 16.1 & \\
\hline Cancer treatment & & & & & & $* * *$ & & & & & & NS \\
\hline Cardiotoxic & 37.3 & 30.3 & 36.5 & 35.4 & 33.2 & & 35.7 & 36.1 & 35.4 & 35.3 & 35.5 & \\
\hline Non-cardiotoxic & 16.8 & 14.7 & 16.7 & 16.7 & 16.0 & & 16.5 & 16.8 & 16.3 & 16.5 & 16.2 & \\
\hline None & 45.9 & 55.0 & 46.8 & 47.8 & 50.9 & & 47.9 & 47.1 & 48.3 & 48.2 & 48.3 & \\
\hline Discordant $\mathrm{PHC}^{\mathrm{g}}$ & & & & & & $* * *$ & & & & & & NS \\
\hline Yes & 35.7 & 43.4 & 39.6 & 43.2 & 47.1 & & 40.7 & 41.0 & 40.9 & 40.9 & 40.8 & \\
\hline No & 64.3 & 56.6 & 60.4 & 56.8 & 52.9 & & 59.3 & 59.0 & 59.1 & 59.1 & 59.2 & \\
\hline Concordant $\mathrm{PHC}^{\mathrm{h}}$ & & & & & & $* * *$ & & & & & & NS \\
\hline Yes & 96.6 & 95.8 & 96.3 & 93.8 & 91.5 & & 95.2 & 95.4 & 95.2 & 95.3 & 95.1 & \\
\hline No & 3.4 & 4.2 & 3.7 & 6.2 & 8.5 & & 4.8 & 4.6 & 4.8 & 4.7 & 4.9 & \\
\hline Mental health condition & & & & & & * & & & & & & NS \\
\hline Yes & 11.9 & 18.8 & 14.1 & 14.6 & 20.5 & & 15.0 & 14.3 & 14.9 & 15.0 & 14.8 & \\
\hline No & 88.1 & 81.2 & 85.9 & 85.4 & 79.5 & & 85.0 & 85.7 & 85.1 & 85.0 & 85.2 & \\
\hline Tobacco use & & & & & & ** & & & & & & NS \\
\hline Yes & 3.4 & 5.3 & 4.3 & 3.5 & 4.7 & & 3.9 & 4.4 & 4.0 & 4.0 & 3.7 & \\
\hline No & 96.6 & 94.7 & 95.7 & 96.5 & 95.3 & & 96.1 & 95.6 & 96.0 & 96.0 & 96.3 & \\
\hline Alcohol use & & & & & & $* * *$ & & & & & & NS \\
\hline Yes & 0.8 & 1.1 & 0.9 & 1.0 & 2.2 & & 0.9 & 1.0 & 1.1 & 1.0 & 0.9 & \\
\hline No & 99.2 & 98.9 & 99.1 & 99.0 & 97.8 & & 99.1 & 99.0 & 98.9 & 99.0 & 99.1 & \\
\hline Primary care visit & & & & & & $* * *$ & & & & & & NS \\
\hline Yes & 83.0 & 89.4 & 85.9 & 84.7 & 86.8 & & 85.2 & 84.7 & 85.3 & 85.4 & 85.3 & \\
\hline No & 17.0 & 10.6 & 14.1 & 15.3 & 13.2 & & 14.8 & 15.3 & 14.7 & 14.6 & 14.7 & \\
\hline Cardiologist visit & & & & & & $* * *$ & & & & & & NS \\
\hline Yes & 68.1 & 73.2 & 71.1 & 59.6 & 64.5 & & 66.8 & 67.6 & 67.2 & 67.4 & 66.7 & \\
\hline No & 31.9 & 26.8 & 28.9 & 40.4 & 35.5 & & 33.2 & 32.4 & 32.8 & 32.6 & 33.3 & \\
\hline SEER region & & & & & & * & & & & & & NS \\
\hline Northeast & 22.9 & 21.4 & 22.8 & 24.6 & 20.5 & & 22.5 & 21.9 & 22.7 & 22.4 & 22.7 & \\
\hline South & 23.4 & 25.8 & 25.5 & 23.4 & 26.6 & & 25.0 & 25.6 & 24.7 & 24.9 & 24.7 & \\
\hline North-Central & 14.8 & 13.1 & 13.8 & 14.4 & 12.3 & & 13.9 & 13.6 & 13.9 & 13.8 & 14.3 & \\
\hline West & 38.8 & 39.7 & 37.9 & 37.6 & 40.5 & & 38.6 & 38.9 & 38.7 & 39.0 & 38.4 & \\
\hline
\end{tabular}

Based on 12,096 elderly FFS Medicare beneficiaries with preexisting CAD and incident breast, colorectal, or prostate cancer diagnosis. Weights were derived using the IPTW approach.

Time-invariant characteristics included cancer diagnosis categories, age, race/ethnicity, concordant and discordant physical health conditions, SMI, SEER region. Time-variant characteristics included tobacco use, alcohol use, depression, anxiety, and routine follow-up with primary care physician and/or cardiologist, and Medicare Part D coverage. Column percentages are reported.

Abbreviations: ACEI, angiotensin-converting enzyme inhibitor; ADH, adherence; ARBs, angiotensin II receptor blockers; CAD, coronary artery disease; FFS, fee-for-service;

IPTW, inverse probability treatment weighting; NS, not significant; PHC, physical health condition; Rx, prescription; Sig, significance; SMI, serious mental illness.

${ }^{a}$ Adherent to both statins and ACEIs/ARBs/ $\beta$-blockers.

${ }^{b}$ Not adherent to both statins and ACEIs/ARBs/ $\beta$-blockers.

'Adherent to either statins or ACEIs/ARBs/B-blockers.

dUse of one medication class and adherent to that class.

e Use of one medication class and not adherent to that medication class.

${ }^{\dagger}$ Asterisks represent significant differences in time-invariant patient-level characteristics based on chi-square tests.

${ }^{9}$ Consisted of arthritis, asthma, chronic obstructive pulmonary disease, osteoporosis, dementia, HIV, and hepatitis. Mental health conditions included anxiety, depression, and SMI (schizophrenia, bipolar disorder, and psychoses).

${ }^{\text {h}}$ Consisted of diabetes, hyperlipidemia, hypertension, stroke, cardiac arrhythmia, and congestive heart failure.

${ }^{*} .01 \leq P<.05 ; * * .001 \leq P<.01 ; * * * P<.001$. 
after IPTW adjustment; after IPTW adjustment, there were no significant differences among other independent variables.

Adherence to both statins and ACEIs/ARBs/ $\beta$ blockers was estimated at $31.2 \%$ during the 120 -day period immediately after cancer diagnosis; $13.7 \%$ were not adherent to both medication classes during the same period. Supplemental eAppendix 2 summarizes the CAD-related hospitalizations over time. The prevalence of CAD-related hospitalizations was $27.4 \%$ during the 120 -day period immediately after cancer diagnosis, which declined to $10.6 \%$ during the last 4 months of the postdiagnosis period.

\section{Associations Between Adherence}

In unadjusted analyses (Table 3), patients not adherent to both medication classes (odds ratio [OR], $1.67 ; 95 \% \mathrm{CI}, 1.58,1.76 ; \mathrm{P}<.0001)$ or those adherent to 1 (out of 2 ) medication class (OR, 1.22; 95\% CI, $1.15,1.30 ; \mathrm{P}<.0001)$ were more likely to have CADrelated hospitalizations compared with those adherent to both medication classes. However, those using and adherent to 1 medication class were significantly less likely to have CAD-related hospitalizations compared with those adherent to both medication classes (OR, 0.76; 95\% CI, 0.66, 0.86; P<.0001).

The adjusted model controlled for time and all other independent variables (Table 3). Findings from the adjusted model were consistent with the unadjusted model. For example, those adherent to both medication classes, those not adherent to both medication classes (adjusted OR [AOR], 1.82; 95\% CI, $1.72,1.92 ; P<.0001$ ) and those adherent to 1 (out of 2) medication class (AOR, 1.26; 95\% CI, 1.17, 1.34; $P<.0001$ ) were more likely to have CAD-related hospitalizations. In contrast to the unadjusted models, those using 1 medication class and not adherent (AOR, 1.20; 95\% CI, 1.09, 1.31; P=.005) were more likely to have CAD-related hospitalizations.

\section{Sensitivity Analyses}

Instrumental Variable Regression: Instrumental variable regression was used to control for unobserved selection bias that can affect medication adherence and the relationship between medication adherence categories and CAD hospitalizations. We selected the percentage of oncologists at the county level as an instrumental variable under the assumption that the effect of oncologists on CAD-related hospitalization occurs only through medication adherence categories. For this analysis, we dichotomized adherence categories into adherent to both medications and other; CAD hospitalization was dichotomized into CAD-related hospitalizations and no CAD hospitalizations, including no CAD and other hospitalizations.

We found that the percent of oncologists at the county level was a strong and valid instrumental variable. The variable oncologists at the county level were significantly associated with medication adherence (OR, 4.27; 95\% CI, 2.15, 8.47; P<.0001) and medication adherence was an endogenous variable (Wald test, $P<.0001$ ). Results from instrumental variable regression revealed that Medicare beneficiaries not adherent to 1 or both medication classes were more likely to have CAD-related hospitalizations compared with those who were adherent to both medication classes $(\beta, 1.20$; SE, $0.10 ; P<.0001)$.

Supplemental eAppendix 3 displays results from adjusted multinomial logistic regressions on CADrelated hospitalization identified from primary diagnosis. Some similarities and differences were present regarding hospitalizations identified from both primary and secondary diagnoses (primary analyses). These findings for hospitalizations identified from primary diagnosis were consistent with the primary analyses (adjusted model), except that no significant difference was observed in those using 1 medication class and adherent to that medication class compared with those adherent to both medication classes.

\section{Associations Between Other Independent Variables and CAD Hospitalization}

Supplemental eAppendix 4 displays results from adjusted generalized estimating equation models with IPTW on adherence for other independent variables. Other variables, such as cancer diagnosis categories, stage, and treatment were significantly associated with CAD-related hospitalization categories.

\section{Discussion}

To the best of our knowledge, this is the first study to examine the association between adherence to statins and ACEIs/ARBs/ $\beta$-blockers on CAD hospitalizations in elderly FFS Medicare beneficiaries with preexisting CAD and incident cancer diagnosis. Consistent with the literature, our results sug- 
CAD Medication Adherence and Hospitalization

\begin{tabular}{|c|c|c|c|c|c|c|}
\hline \multirow[b]{2}{*}{ Variables } & \multicolumn{3}{|c|}{ CAD-Related Hospitalization } & \multicolumn{3}{|c|}{ Other Hospitalization } \\
\hline & OR & $95 \% \mathrm{Cl}$ & Sig $^{\mathrm{a}}$ & OR & $95 \% \mathrm{Cl}$ & Sig $^{\mathrm{a}}$ \\
\hline \multicolumn{7}{|l|}{ Unadjusted analysis ${ }^{b}$} \\
\hline \multicolumn{7}{|l|}{ Medication adherence categories } \\
\hline No $A D H 2 R x^{c}$ & 1.67 & $(1.58,1.76)$ & $* * *$ & 1.88 & $(1.75,2.00)$ & *** \\
\hline ADH 1 of $2 R x^{d}$ & 1.22 & $(1.15,1.30)$ & $* * *$ & 1.36 & $(1.25,1.46)$ & $* * *$ \\
\hline$A D H 1 R x^{e}$ & 0.76 & $(0.66,0.86)$ & $* * *$ & 1.59 & $(1.48,1.70)$ & $* * *$ \\
\hline No ADH $1 R^{f}$ & 1.05 & $(0.94,1.15)$ & & 2.10 & $(1.99,2.22)$ & $* * *$ \\
\hline$A D H 2 R x^{g}$ & & $\operatorname{Ref}^{h}$ & & & $\operatorname{Ref}^{h}$ & \\
\hline \multicolumn{7}{|l|}{ Cancer diagnosis categories } \\
\hline Women with breast cancer & 1.15 & $(1.07,1.23)$ & $* * *$ & 1.61 & $(1.52,1.70)$ & $* * *$ \\
\hline Women with colorectal cancer & 3.39 & $(3.31,3.47)$ & $* * *$ & 4.23 & $(4.14,4.32)$ & $* * *$ \\
\hline Men with colorectal cancer & 3.60 & $(3.52,3.68)$ & $* * *$ & 2.91 & $(2.80,3.02)$ & *** \\
\hline Men with prostate cancer & & $\operatorname{Ref}^{h}$ & & & $\operatorname{Ref}^{h}$ & \\
\hline \multicolumn{7}{|l|}{ Cancer stage } \\
\hline $0 / 1$ & 0.93 & $(0.87,0.99)$ & * & 0.82 & $(0.77,0.88)$ & $* * *$ \\
\hline III/IV & 2.04 & $(1.97,2.12)$ & $* * *$ & 2.31 & $(2.22,2.40)$ & $* * *$ \\
\hline II & & $\operatorname{Ref}^{h}$ & & & $\operatorname{Ref}^{h}$ & \\
\hline \multicolumn{7}{|l|}{ Cancer treatment } \\
\hline Cardiotoxic cancer treatment & 1.30 & $(1.16,1.44)$ & $* * *$ & 2.36 & $(2.16,2.56)$ & $* * *$ \\
\hline None & 0.88 & $(0.76,1.00)$ & * & 1.11 & $(0.93,1.29)$ & \\
\hline Non-cardiotoxic & & $\operatorname{Ref}^{h}$ & & & $\operatorname{Ref}^{h}$ & \\
\hline Variables & AOR & $95 \% \mathrm{Cl}$ & Sig & AOR & $95 \% \mathrm{Cl}$ & Sig \\
\hline \multicolumn{7}{|l|}{ Adjusted analysis ${ }^{b}$} \\
\hline \multicolumn{7}{|l|}{ Medication adherence categories } \\
\hline No ADH $2 R^{c}$ & 1.82 & $(1.72,1.92)$ & $* * *$ & 2.00 & $(1.87,2.13)$ & $* * *$ \\
\hline ADH 1 of $2 R x^{d}$ & 1.26 & $(1.17,1.34)$ & $* * *$ & 1.39 & $(1.28,1.49)$ & $* * *$ \\
\hline $\mathrm{ADH} 1 \mathrm{Rx} \mathrm{e}^{\mathrm{e}}$ & 0.80 & $(0.70,0.90)$ & $* * *$ & 1.65 & $(1.54,1.76)$ & *** \\
\hline No $A D H 1 R^{f}$ & 1.20 & $(1.09,1.31)$ & ** & 2.34 & $(2.22,2.46)$ & *** \\
\hline ADH $2 R^{9}$ & $\operatorname{Ref}^{h}$ & & & $\operatorname{Ref}^{\text {h }}$ & & \\
\hline \multicolumn{7}{|l|}{ Cancer diagnosis categories } \\
\hline Women with breast cancer & 1.18 & $(1.08,1.29)$ & $\star *$ & 1.59 & $(1.47,1.71)$ & $* * *$ \\
\hline Women with colorectal cancer & 3.31 & $(3.20,3.42)$ & $* * *$ & 4.20 & $(4.07,4.33)$ & $* * *$ \\
\hline Men with colorectal cancer & 3.78 & $(3.67,3.88)$ & $* * *$ & 2.80 & $(2.67,2.93)$ & *** \\
\hline Men with prostate cancer & $\operatorname{Ref}^{h}$ & & & & $\operatorname{Ref}^{h}$ & \\
\hline \multicolumn{7}{|l|}{ Cancer stage } \\
\hline $0 / 1$ & 0.65 & $(0.55,0.74)$ & $* * *$ & 0.71 & $(0.60,0.81)$ & $* * *$ \\
\hline III/IV & 1.25 & $(1.15,1.34)$ & $* * *$ & 1.37 & $(1.26,1.47)$ & $* * *$ \\
\hline ॥ & & $\operatorname{Ref}^{h}$ & & & $\operatorname{Ref}^{h}$ & \\
\hline \multicolumn{7}{|l|}{ Cancer treatment } \\
\hline Cardiotoxic & 1.31 & $(1.14,1.49)$ & ** & 2.14 & $(1.91,2.37)$ & *** \\
\hline None & 0.84 & $(0.69,0.98)$ & * & 1.07 & $(0.86,1.28)$ & \\
\hline Non-cardiotoxic & & $\operatorname{Ref}^{h}$ & & & $\operatorname{Ref}^{h}$ & \\
\hline
\end{tabular}

Based on 12,096 elderly fee-for-service Medicare beneficiaries with preexisting CAD and incident breast, colorectal, or prostate cancer diagnosis. Weights were derived

using the IPTW approach. education, concordant and discordant physical health conditions, serious mental illness, SEER region, and county-level characteristics. Time-variant characteristics included tobacco use, alcohol use, depression, anxiety, and routine follow-up with primary care physician and/or cardiologist, and Medicare Part D coverage.

Abbreviations: ACEls, angiotensin-converting enzyme inhibitors; $A D H$, adherence; $A O R$, adjusted odds ratio; $A R B$, angiotensin II receptor blockers; $C A D$, coronary artery

disease; GEE, generalized estimating equations; IPTW, inverse probability treatment weighting; OR, odds ratio; Rx, prescription; Sig, significance.

${ }^{a}$ Asterisks represent significant differences in medication adherence groups, derived from multinomial logistic regression models with repeated measures.

b ORs are reported for unadjusted GEE model and AORs are reported for adjusted GEE model.

${ }^{c}$ Not adherent to both statins and ACEIs/ARBs/ $\beta$-blockers.

${ }^{\mathrm{d}}$ Adherent to either statins or ACEIs/ARBs/ $\beta$-blockers.

eUse of one medication class and adherent to that class.

fUse of one medication class and not adherent to that medication class.

${ }^{9}$ Adherent to both statins and ACEIs/ARBs/ $\beta$-blockers.

${ }^{\text {h}}$ Reference group is patients with no hospitalization.

${ }^{*} .01 \leq P<.05 ;{ }^{* *} .001 \leq P<.01 ; * * * P<.001$. 
gest that those adherent to both statins and ACEIs/ ARBs/ $\beta$-blockers or nonadherent to either both or single medication classes were more likely to have CAD-related hospitalizations. Nonadherence to statins and ACEIs/ARBs/ $\beta$-blockers has been reported to have negative cardiovascular health consequences, including cardiovascular events and hospitalization. ${ }^{18,20,38}$

Counterintuitively, our study findings indicated that those using a single medication class and adherent to that medication class were less likely to have CAD-related hospitalizations compared with those adherent to both medication classes. We speculate plausible reasons for this finding. We did not control for CAD severity with the "gold standard measure," therefore, the lower likelihood of hospitalization by those with single medication class may partially reflect less-severe CAD. It is also plausible that individuals on a single class of medication may be adhering to Life's Simple 7 steps recommended by the American Heart Association: (1) managing blood pressure, (2) controlling cholesterol, (3) controlling blood sugar, (4) being active, (5) eating a healthy diet, (6) maintaining normal weight, and (7) not smoking. ${ }^{39}$ Unfortunately, none of these variables were available in our database.

Consistent with findings from a previous study on patients diagnosed with prostate cancer, ${ }^{7}$ we observed that CAD-related hospitalizations increased in the period immediately after cancer diagnosis for all medication adherence categories. It is possible that cardiotoxic cancer treatments increase the risk of CAD-related hospitalizations. ${ }^{9-11}$ Our study findings showed that those receiving cardiotoxic cancer treatment were more likely to have CAD-related hospitalizations.

A meta-analysis of clinical trials showed that the prophylactic use of $\beta$-blockers, ACEIs, ARBs, or statins reduced the risk of newly developed heart failure in patients administered anthracycline. ${ }^{40}$ Our study findings revealed that the interaction effect between adherence to both medication classes and cardiotoxic cancer treatment was not statistically significant. It is possible that the established CAD diminishes the cardioprotective effect of these medications. In addition, the meta-analysis did not specifically focus on the elderly population and the specific cancers included in our study. Further studies with other characteristics, such as physical activ- ity, complementary medicine, and polypharmacy, are needed to provide conclusive evidence on the lack of interaction between adherence and cardiotoxic cancer treatment.

\section{Clinical and Policy Implications}

Both CAD and cancer are life-threatening conditions and co-management of both conditions can be challenging. However, lack of effective management of CAD may not only increase related complications but also can have negative consequences on cancer prognosis. Current clinical practices recommend stabilizing preexisting CAD before initiating cancer treatment. For example, appropriate pharmacologic management (statins and $\beta$-blockers) of patients with preexisting CAD is recommended prior to cancer surgery. ${ }^{41}$ Given the complexity of interaction between CAD and cancer, it is important to routinely monitor medication adherence in general clinical practice and provide linkages to support services that can increase medication adherence. This may warrant integration of cardiovascular care in elderly patients diagnosed with incident cancer. An example of integrated care is the cardio-oncology clinics that provide an interdisciplinary and integrative management approach to patients with cardiovascular risks or conditions. ${ }^{42}$

It is possible that certain CAD-related hospitalizations in our study may be readmissions or preventable hospitalizations, which have policy implications. The Hospital Readmissions Reduction Program, under the Affordable Care Act, provided CMS the authority to reduce payments to certain hospital readmissions that were deemed avoidable..$^{43}$ The emphasis was on Medicare readmissions related to heart failure, myocardial infarction, and pneumonia. Reducing avoidable readmissions can enhance patient quality of care and lower healthcare spending. In addition, based on the Agency for Healthcare Research and Quality's (AHRQ's) prevention quality indicators, hospitalizations related to conditions, such as angina without procedure and congestive heart failure, are considered preventable hospitalizations with good outpatient care. Therefore, it is important to implement interventions for improving medication adherence and to have integrated care for patients with preexisting CAD and incident cancer diagnosis for reducing avoidable hospitalizations. 


\section{Strengths and Limitations}

Our study findings need to be interpreted in the context of its limitations. First, the study findings cannot be generalized to all Medicare beneficiaries because the study population is restricted to those aged $\geq 68$ years and with continuous enrollment in Medicare Parts A, B, and D. It must be noted that not all Medicare beneficiaries were enrolled in Part D plans. Further, the population was restricted to those with breast, colorectal, or prostate cancer and preexisting CAD. Second, we were not able to control for many other variables, such as lifestyle health behaviors, knowledge, attitude, preferences, and other factors that may affect adherence, as well as hospitalizations. ${ }^{44}$ However, we controlled for a comprehensive list of variables to examine multivariable associations. We used a proxy measure for CAD severity, which may not reflect the precise CAD severity in the population. Gold standards for measuring CAD severity, such as stress tests, are not available in the Medicare database. Third, the migration of patients in and out of SEER registry geographic catchment areas makes it difficult to measure outcomes dependent on long-term follow-up, which leads to undercounting of the study population. Further, it is possible that CAD diagnosis may be undercoded or misclassified in claims data, as these data are dependent on professional ICD coding. Therefore, CAD-related diagnosis and CAD-related hospitalizations may be underestimated. Fourth, the need factor is a social construct and comprises perceived and evaluated need in the Andersen model. Evaluated need is measurable, whereas perceived need is partly determined by health beliefs. This study included "evaluated need" as it comprised measurable/objective factors that were available in the SEER-Medicare database.
Lastly, we used prescription claims for measuring adherence. Because the Part D file contains only filled prescriptions, it is not known whether the patients actually used the medications or adhered to their providers' instructions.

Despite these limitations, our study adds to the nascent literature on cardio-oncology and has several strengths. First, the current study included a large cohort of patients over a long period across a variety of providers and focused on real-world practice patterns. Second, the study used a robust repeated measure design. Third, the study used a variety of statistical methods to control for both observed and unobserved selection bias to ensure robustness of findings.

\section{Conclusions}

Nonadherence to both statins and ACEIs/ARBs/ $\beta$ blockers can increase the risk other CAD-related hospitalizations in preexisting CAD and incident breast, colorectal, or prostate cancer. Both nonadherence and hospitalizations are affected by a multitude of factors, comprising patient-related, clinical (disease- and therapy-related), socioeconomic, and healthcare system aspects. Understanding how and to what extent these factors affect nonadherence and hospitalization may shed light on the clinical practice and policy implementation. Our study included a comprehensive list of factors; however, it is limited by the scope of the SEER-Medicare linked database. Future studies need to examine the role of patient-related factors (lifestyle health behaviors, knowledge, attitude, and preferences) that were not measured in this study so that strategies to improve adherence in this population can be developed.

\section{References}

1. QuickStats: Rate of Hospitalization for Coronary Heart Disease, by Age Group — National Hospital Discharge Survey, United States, 2000-2010. Available at: http://www.cdc.gov/mmwr/preview/mmwrhtml/mm6123a5. htm. Accessed November 20, 2016.

2. Luepker RV. Falling coronary heart disease rates: a better explanation? Circulation 2016;133:8-11.

3. Krumholz HM, Normand SL, Wang Y. Trends in hospitalizations and outcomes for acute cardiovascular disease and stroke, 1999-2011. Circulation 2014;130:966-975.

4. HCUPnet. Statistics on Hospital Stays. Available at: http://hcupnet.ahrq gov/. Accessed November 23, 2016

5. Russell MW, Huse DM, Drowns S, et al. Direct medical costs of coronary artery disease in the United States. Am J Cardiol 1998;81:1110-1115.

6. Mozaffarian D, Benjamin EJ, Go AS, et al. Heart disease and stroke statistics-2015 update: a report from the American Heart Association. Circulation 2015;131:e29-322.

7. Raval AD, Madhavan S, Mattes MD, et al. Impact of prostate cancer diagnosis on noncancer hospitalizations among elderly Medicare beneficiaries with incident prostate cancer. J Natl Compr Canc Netw 2016;14:186-194.

8. Zoller B, Ji J, Sundquist J, Sundquist K. Risk of coronary heart disease in patients with cancer: a nationwide follow-up study from Sweden. Eur J Cancer 2012;48:121-128.

9. Yusuf SW, Razeghi P, Yeh ET. The diagnosis and management of cardiovascular disease in cancer patients. Curr Probl Cardiol 2008;33:163196.

10. Herrmann J, Lerman A, Sandhu NP, et al. Evaluation and management of patients with heart disease and cancer: cardio-oncology. Mayo Clin Proc 2014;89:1287-1306. 
11. Accordino MK, Neugut AI, Hershman DL. Cardiac effects of anticancer therapy in the elderly. J Clin Oncol 2014;32:2654-2661.

12. Darby SC, Ewertz M, McGale P, et al. Risk of ischemic heart disease in women after radiotherapy for breast cancer. N Engl J Med 2013;368:987998.

13. McGale P, Darby SC, Hall P, et al. Incidence of heart disease in 35,000 women treated with radiotherapy for breast cancer in Denmark and Sweden. Radiother Oncol 2011;100:167-175.

14. Onwudiwe NC, Kwok Y, Onukwugha E, et al. Cardiovascular event-free survival after adjuvant radiation therapy in breast cancer patients stratified by cardiovascular risk. Cancer Med 2014;3:1342-1352.

15. Chen J, Long JB, Hurria A, et al. Incidence of heart failure or cardiomyopathy after adjuvant trastuzumab therapy for breast cancer. J Am Coll Cardiol 2012;60:2504-2512.

16. Yood MU, Wells KE, Alford SH, et al. Cardiovascular outcomes in women with advanced breast cancer exposed to chemotherapy. Pharmacoepidemiol Drug Saf 2012;21:818-827.

17. Chavez-MacGregor M, Zhang N, Buchholz TA, et al. Trastuzumab-related cardiotoxicity among older patients with breast cancer. J Clin Oncol 2013;31:4222-4228.

18. Chowdhury R, Khan H, Heydon E, et al. Adherence to cardiovascular therapy: a meta-analysis of prevalence and clinical consequences. Eur Heart J 2013;34:2940-2948.

19. Ho PM, Magid DJ, Masoudi FA, et al. Adherence to cardioprotective medications and mortality among patients with diabetes and ischemic heart disease. BMC Cardiovasc Disord 2006;6:48.

20. Bitton A, Choudhry NK, Matlin OS, et al. The impact of medication adherence on coronary artery disease costs and outcomes: a systematic review. Am J Med 2013;126:357.e7-357.e27.

21. Hindler K, Cleeland CS, Rivera E, Collard CD. The role of statins in cancer therapy. Oncologist 2006;11:306-315.

22. Cardwell CR, Hicks BM, Hughes C, Murray LJ. Statin use after colorectal cancer diagnosis and survival: a population-based cohort study. J Clin Oncol 2014;32:3177-3183.

23. $\mathrm{Yu} O$, Eberg M, Benayoun $\mathrm{S}$, et al. Use of statins and the risk of death in patients with prostate cancer. J Clin Oncol 2014;32:5-11.

24. Ahern TP, Lash TL, Thwin SS, Silliman RA. Impact of acquired comorbidities on all-cause mortality rates among older breast cancer survivors. Med Care 2009;47:73-79

25. Coebergh JW, Janssen-Heijnen ML, Post PN, Razenberg PP. Serious comorbidity among unselected cancer patients newly diagnosed in the southeastern part of The Netherlands in 1993-1996. J Clin Epidemiol 1999;52:1131-1136.

26. de Rijke JM, Schouten LJ, Hillen HF, et al. Cancer in the very elderly Dutch population. Cancer 2000;89:1121-1133.

27. Lloyd-Jones DM, Larson MG, Beiser A, Levy D. Lifetime risk of developing coronary heart disease. Lancet 1999;353:89-92.

28. Andersen RM. Revisiting the behavioral model and access to medical care: does it matter? J Health Soc Behav 1995;36:1-10.
29. Andersen R, Newman JF. Societal and individual determinants of medical care utilization in the United States. Milbank Mem Fund Q Health Soc 1973;51:95-124

30. Engels EA, Pfeiffer RM, Ricker W, et al. Use of Surveillance, Epidemiology, and End Results-Medicare data to conduct case-control studies of cance among the US elderly. Am J Epidemiol 2011;174:860-870.

31. American Community Survey. Summary File Data. United States Census Bureau Web site. Available at: https://www.census.gov/programs-surveys/ acs/data.html. Accessed March 30, 2016.

32. Area Health Resource Files. HRSA Data Warehouse Web site. Available at: http://ahrf.hrsa.gov/overview.htm. Accessed March 2, 2016.

33. Chronic Conditions Data Warehouse. Available at: https://www.ccwdata. org/web/guest/condition-categories. Accessed May 18, 2016.

34. Greer SA, Nwaise IA, Casper ML. Atlas of Heart Disease Hospitalizations Among Medicare Beneficiaries. Atlanta, GA: U.S. Department of Health and Human Services, Centers for Disease Control and Prevention; 2010.

35. Choudhry NK, Shrank WH, Levin RL, et al. Measuring concurrent adherence to multiple related medications. Am J Manag Care 2009; 15:457-464

36. Evaluation of the CMS-HCCRisk Adjustment Model. Available at: https:// www.cms.gov/Medicare/Health-Plans/MedicareAdvtgSpecRateStats/ downloads/Evaluation_Risk_Adj_Model_2011.pdf. Accessed March 2, 2016.

37. Hubbard AE, Ahern J, Fleischer NL, et al. To GEE or not to GEE: comparing population average and mixed models for estimating the associations between neighborhood risk factors and health. Epidemiology 2010;21:467-474.

38. Wald NJ, Law MR. A strategy to reduce cardiovascular disease by more than $80 \%$. BMJ 2003;326:1419.

39. My Life Check - Life's Simple 7. American Heart Association Web site. Available at: http://www.heart.org/HEARTORG/Conditions/My-Life Check..-Lifes-Simple-7 UCM_471453_Article.jsp\#.WS-FtfDyuUk. Accessed May 25, 2017.

40. Kalam K, Marwick TH. Role of cardioprotective therapy for prevention of cardiotoxicity with chemotherapy: a systematic review and meta-analysis. Eur J Cancer 2013;49:2900-2909.

41. Krone RJ. Managing coronary artery disease in the cancer patient. Prog Cardiovasc Dis 2010;53:149-156.

42. Clinical Updates: Cardio-Oncology Clinics Integrate Specialty Clinical Care. Mayo Clinic Web site. Available at: http://www.mayoclinic.org/ medical-professionals/clinical-updates/cardiovascular/cardio-oncologyclinics-integrate-specialty-clinical-care. Accessed November 22, 2016.

43. Mark TL, Tomic KS, Kowlessar N, et al. Hospital readmission among medicaid patients with an index hospitalization for mental and/or substance use disorder. J Behav Health Serv Res 2013;40:207-221.

44. Carroll AE. The Power of Simple Life Changes to Prevent Heart Disease. Available at: http://www.nytimes.com/2016/12/12/ upshot/the-power-of-simple-life-changes-to-prevent-heart-disease. $\mathrm{html}$ ?contentCollection $=$ smarter-living\& $\&$ $\&$ action $=$ click\& pgtype $=\mathrm{Ho}$ mepage $\&$ clickSource $=$ story-heading $\&$ module $=$ second-column-region $\&$ $\mathrm{r}=0$. Accessed December 22, 2016. 